\title{
UV habitability and dM stars: an approach for evaluation of biological survival
}

\author{
Ximena C. Abrevaya ${ }^{1,2}$, Eduardo Cortón ${ }^{2}$ and Pablo J. D. Mauas ${ }^{1}$ \\ ${ }^{1}$ Institute for Astronomy and Space Physics UBA-CONICET. \\ Buenos Aires, Argentina. \\ email: abrevaya@iafe.uba.ar \\ ${ }^{2}$ Biochemistry Department, Faculty of Exact Sciences, UBA. \\ Buenos Aires, Argentina.
}

\begin{abstract}
Dwarf M stars comprise about 75 percent of all stars in the galaxy. For several years planets orbiting $M$ stars have been discarded as suitable places for development of life. This paradigm now has changed and terrestrial-type planets within liquid-water habitable zones (LW-HZ) around M stars are reconsidered as possible hosts for life as we know it. Nevertheless, large amount of UV radiation is emitted during flares by this stars, and it is uncertain how these events could affect biological systems. In particular UV-C $(\lambda<290 \mathrm{~nm})$ exhibits the most damaging effects for living organisms. To analyze the hypothesis that UV could set a limit for the development of extraterrestrial life, we studied the effect of UV-C treatment on halophile archaea cultures. Halophile archaea are extremophile organisms, they are exposed to intense solar UV radiation in their natural environment so they are generally regarded as relatively UV tolerant. Halophiles inhabits in hipersaline environments as salt lakes but also have been found in ancient salt deposits as halites and evaporites on Earth. Since evaporites have been detected in Martian meteorites, these organisms are proposed as plausible inhabitants of Marslike planets. Our preliminary results show that even after UV damage, the surviving cells were able to resume growth with nearly normal kinetics.
\end{abstract}

Keywords. astrobiology, stars: flare, ultraviolet: stars, methods: laboratory

\section{Introduction}

According to "Mediocrity principle", which proposes that our planetary system, life on Earth, and technological civilizations are an average case in the Universe (von Hoerner, 1961), life as we know it could emerge in any place with conditions similar to Earth, and would develop following similar selection rules.

The Habitability Criteria define which places are appropriate for life according to physical and chemical environmental factors (Cockell, 2007). Usually, the presence of liquid water in the planet's surface which is known as Liquid-Water Habitable Zone (LWHZ)is the most important aspect for habitability (Huang, 1959; Dole, 1964; Hart, 1979). However, other important environmental factors as UV radiation can play a significant role in habitability. It is well known that UV have deleterious effects for cells, acting as a genotoxic agent and inducting DNA damage, or damaging a wide variety of proteins and lipids. In particular, high exposure to UV-C $(\lambda<290 \mathrm{~nm})$ is lethal to most terrestrial biological systems. Therefore, UV radiation should also be considered as an habitability criteria (Buccino et al., 2006).

In particular, during the last decade there was a change in paradigm, which led to consider that terrestrial planets around dM stars can be suitable places for the emergence 
and evolution of life. However, many dMe stars emit large amount of UV radiation during flares, and it is uncertain how these UV events can affect life (Buccino et al., 2007).

Extremophile organisms are subject of study in astrobiology due to their capacity to survive in extreme physicochemical environmental conditions. Between these group there is a kind of microorganisms which inhabits in environments with high salt concentrations (3.4 - 5.1 M NaCl range for optimally growth), commonly known as halophilic archaea. These halophiles inhabits in hipersaline environments as salt lakes but also have been found in ancient salt deposits as halites (salt sediments several million years old) and in other ancient deposits as evaporites or rock salt (Whitby et al. 2000; Fendrihan et al., 2006). Since evaporites have been detected in Martian SNC meteorites (Gooding, 1992; Rieder et al., 2004), these microorganisms are proposed as plausible inhabitants of Marslike planets or other extrasolar planets (Stan-Lotter et al., 2004). Moreover, because much halophiles are exposed to intense solar UV radiation in their natural environment, so they are generally regarded as relatively UV tolerant. In this work we examine the effect of UV-C on Natrialba magadii an halophile archaea initially isolated from Magadi Lake in Kenya, Africa.

\section{Methods}

Cultures of $N$. magadii were grown around optical density $(O D)$ related to midexponential phase. Samples were divided in five groups: Control (non irradiated culture), and irradiated for 5, 10, 20 and 30 minutes (doses: 1110, 2220, 4440, $6660 \mathrm{~J} \mathrm{~m}^{-2}$, respectively). Liquid culture was diluted to reach $O D_{600 \mathrm{~nm}}=0.05$, and irradiated being exposed to a UV-C source (Phillips $15 \mathrm{~W} \mathrm{Hg}$ lamp $\lambda=254 \mathrm{~nm}$, irradiance $3.7 \mathrm{~W} \mathrm{~m} \mathrm{~m}^{-2}$ ). Aliquots of the irradiated and control groups were withdrawn after different irradiation times and the effect of the UV treatment was assesed following the changes of the growth kinetics.

\section{Results}

To obtain growth curves, OD values for each sample were acquired at different times after irradiation and were plotted versus post-irradiation time. Then data points were fitted to a modified logistic equation (A. Mendez, personal communication), as is shown:

$$
y=\frac{A}{1+\exp \left[\frac{4 \mu_{m}}{A}(\lambda-t)+2\right]}
$$

where $y$ is proportional to biomass (in our case OD), $A$ is the maximum growth, $\mu_{m}$ is the maximum specific growth rate, $\lambda$ is the lag time, and $t$ is the post-irradiation time (see Fig. 1.a). Growth curves were obtained for each dose assayed as shown in Figure 1.b and parameters were calculated according to equation 1 . Our results show that there is a dose dependent delay in the growth of the different samples (Table 1).

\begin{tabular}{lcccc}
\hline Experimental group & $\begin{array}{c}\text { Irradiance } \\
W m^{-2}\end{array}$ & $\begin{array}{c}\text { Irradiation time } \\
\mathrm{s}\end{array}$ & $\begin{array}{c}\text { Dose } \\
\mathrm{Jm}^{-2}\end{array}$ & $\begin{array}{c}\text { Lag time }(\lambda) \\
h\end{array}$ \\
\hline Control & 0 & 0 & 0 & $20.13 \pm 2.36$ \\
UV 5 min & 3.7 & 300 & 1110 & $20.31 \pm 2.41$ \\
UV 10 min & 3.7 & 600 & 2220 & $27.95 \pm 1.50$ \\
UV 20 min & 3.7 & 1200 & 4440 & $34.35 \pm 1.52$ \\
UV 30 min & 3.7 & 1800 & 6660 & $48.24 \pm 2.07$ \\
\hline
\end{tabular}

Table 1. Parameters obtained from the experimental data 

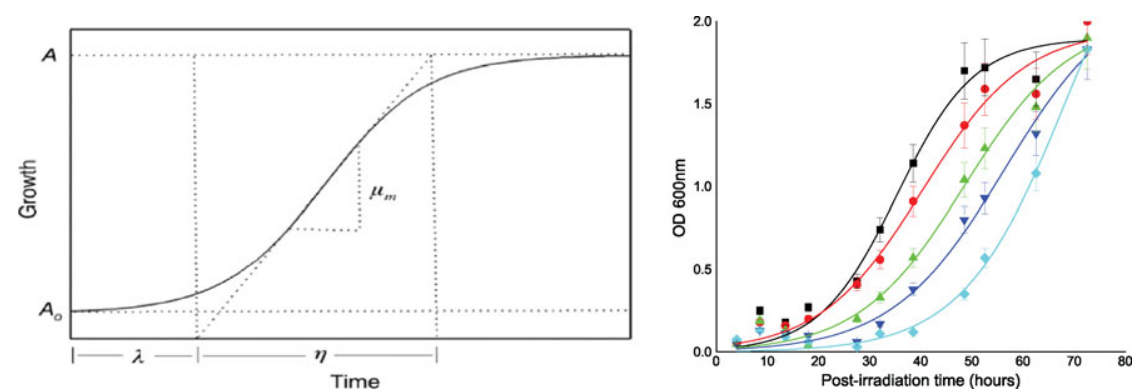

Figure 1. (a) : Growth curve showing the meaning of the parameters in equation 1, where " $A_{o}$ " is the initial growth and " $\eta$ " is the growth time. (b) : Growth curves for all samples. Each line shows different UV doses: control, $0 \mathrm{~J} / \mathrm{m}^{2}(\boldsymbol{\square})$ and irradiated $1110 \mathrm{~J} / \mathrm{m}^{2}(\bullet) ; 2220 \mathrm{~J} / \mathrm{m}^{2}(\boldsymbol{\Delta}) ; 4440 \mathrm{~J} / \mathrm{m}^{2}(\mathbf{\nabla}) ; 6660 \mathrm{~J} / \mathrm{m}^{2}(\bullet)$.

\section{Discussion}

It is well known that UV induces diverse kind of damage in cells. In particular UV$\mathrm{C}$ is a potent inductor of DNA chemical modifications like formation of cyclobutane pyrimidine dimers and 6-4 photoproducts (Mitchell et al., 1989; Cadet et al., 2005). If these alterations are not repaired they can cause blockage of diverse cellular processes, leading to mutations or cell death. Growth delay could be explained by some of these effects, and could be related to possible sublethal effects like cell cycle arrest (Rudolph et al., 2007) induced by DNA damage or by other processes capable of blocking cell growth like tRNA photodamage (Opezzo \& Pizarro, 2001). In such cases bacterial population remains in lag phase, which is visualized as a dose dependent displacement of the growth curve. In any case these preliminary results show that even after significant UV damage, as judged by the absence of detectable growth for more than 30 hours, the surviving cells were able to resume growth with nearly normal kinetics at all doses analyzed. UV irradiances used in the present work are of the same order to those received from a weak flare, such as those detected in EV-Lac $\left(4.94 \mathrm{Wm}^{-2}\right)$. In next experiments we will include as an important factor the flare frequency which usually is very high: e.g., one flare every 10 hours for Proxima Centauri and every 1-1.5 hours for AD Leo (Cincunegui et al., 2007; Mauas \& Falchi, 1994).

\section{References}

Buccino, A. P., Lemarchand, G. A., \& Mauas, P. J. D. 2007, Icarus, 192, 582

Buccino, A. P., Lemarchand, G. A., \& Mauas, P. J. D. 2006, Icarus, 183, 491

Cadet, J., Sage, E., \& Douki, T. 2005, Mutat.Res, 571, 3

Cincunegui, C., Diaz, R. F., \& Mauas, P. J. D. 2007, aap,461, 1107

Cockell, C. S. 2007, in: Horneck, G., \& Rettberg, P., Eds. C. C. in Astrob. (Wiley-VCH)151, 177

Dole, S. H. 1964, Habitable planets for man (Blaisdell Pub. Co., 1st ed.), New York

Fendrihan, S., et al. 2006, Rev.Environ.Sci.Biotechnol, 5, 203

Gooding, J. L. 1992, Icarus, 99, 28

Hart, M. H. 1979, Icarus, 37, 351

Huang, S. S. 1959, Am.Sci., 47, 397

Mauas, P. J. D. \& Falchi, A. 1994, aap, 281, 129

Mitchell, D. L. \& Nairn, R. S. 1989, Photochem.photobiol., 49, 805

Opezzo, O. J. \& Pizarro, R. A. 2001, J.Photochem.Photobiol.B:Biology, 62, 158

Rieder, R., et al. 2005, Science, 306, 1746

Rudolph, C. J., Upton, A. L., \& Lloyd, R. 2007, Genes EBDev., 21, 668

Stan-Lotter, H., et al. 2004, Halophilic microorganisms, (Springer Verlag, N.Y.), 89, 102

von Hoerner, S. 1961, Science 134, 1839

Whitby, J., et al. 2000, Science, 288, 1819 